\title{
Proliferación Microbiana y Calidad Posdescongelación de Semen Equino Criopreservado en Presencia de Antibióticos
}

\author{
Microbial Growth and Post-thaw Quality of Stallion Semen Cryopreserved \\ in Presence of Antibiotics \\ Giovanni Restrepo Betancur ${ }^{1,3}$, Leonardo Rodríguez Mazo², \\ Juan Esteban Duque Cortés ${ }^{2}$
}

\section{Resumen}

\begin{abstract}
Los antibióticos son utilizados en la criopreservación de semen equino con el fin de controlar la proliferación de microorganismos; sin embargo, estos pueden tener efectos negativos sobre los espermatozoides. La presente investigación tuvo como objetivo evaluar el efecto de dos preparaciones antibióticas sobre el desarrollo microbiano y la calidad de semen equino criopreservado. El semen de 10 caballos criollos colombianos fue criopreservado por congelación convencional, con dos preparaciones antibióticas: P1 (penicilina $10000 \mathrm{UI} / \mathrm{ml}$ y estreptomicina $10 \mathrm{mg} / \mathrm{ml}$ ) y P2 (penicilina $10000 \mathrm{UI} / \mathrm{ml}$, estreptomicina $10 \mathrm{mg} / \mathrm{ml}$ y anfotericina B $25 \mu \mathrm{g} / \mathrm{ml}$ ) y un grupo control sin antibióticos. Posterior a la descongelación, se realizó la evaluación computarizada de la movilidad espermática, la prueba hipoosmótica (HOS) y el conteo de bacterias y hongos en el semen. El análisis estadístico se realizó mediante modelos lineales generalizados (GLM) y la comparación de medias por Tukey. Se encontró diferencia significativa $(\mathrm{p}<0.05)$ para movilidad total (MT\%) entre P1 (32.2 \pm 17.6$)$ y P2 (34.4 \pm 19.1$)$ con el grupo control (39.7 $\pm 16.5)$ y para movilidad progresiva (MP\%) entre P1 $(15.5 \pm 14.8)$ con P2 $(17.9 \pm 16.5)$ y el control (19.6 \pm 14.1$)$. Para la proliferación microbiana se encontró diferencia significativa $(\mathrm{p}<0.05)$ para bacterias (UFC/ml) entre P1 $(167.7 \pm 237.1)$ y P2 $(240.1 \pm 321.0)$ con el grupo control (464.4 \pm 448.5 ). El uso de preparaciones antibióticas con penicilina, estreptomicina y anfotericina $B$ en la criopreservación de semen equino reduce la proliferación de bacterias, pero produce una disminución en la movilidad espermática posdescongelación.
\end{abstract}

Palabras clave: bacterias, calidad seminal, congelación, hongos

${ }^{1}$ Facultad de Ciencias Agrarias, Universidad Nacional de Colombia, Sede Medellín, Medellín, Medellín, Colombia

${ }^{2}$ Facultad de Ciencias Agrarias, Politécnico Colombiano Jaime Isaza Cadavid, Medellín, Colombia

${ }^{3}$ Email: grestre0@unal.edu.co

Recibido: 18 de agosto de 2015

Aceptado para publicación: 26 de noviembre de 2015 
Antibiotics are used in cryopreservation of stallion semen in order to control the growth of microorganisms; however they can have negative effects on the spermatozoa. This study aimed to evaluate the effect of two antibiotic preparations on microbial growth and quality of cryopreserved stallion semen. Semen from 10 Colombian Creole horses was cryopreserved by conventional freezing with two antibiotic preparations: P1 (penicillin $10000 \mathrm{UI} / \mathrm{ml}$ and streptomycin $10 \mathrm{mg} / \mathrm{ml}$ ) and P2 (penicillin $10000 \mathrm{UI} / \mathrm{ml}$, streptomycin 10 $\mathrm{mg} / \mathrm{ml}$, and amphotericin B $25 \mu \mathrm{g} / \mathrm{ml}$ ) and a control group without antibiotics. After thawing, computerized sperm motility evaluation, hypoosmotic test (HOS) and counting of bacteria and fungi in semen were performed. Statistical analysis was done using generalized linear models (GLM) and comparison of means by Tukey. Significant difference $(\mathrm{p}<0.05)$ for total motility $(\mathrm{MT} \%)$ between P1 $(32.2 \pm 17.6)$ and P2 $(34.4 \pm 19.1)$ with the control group $(39.7 \pm 16.5)$ was found, as well as for progressive motility (MP\%) between $\mathrm{P} 1(15.5 \pm 14.8)$ with $\mathrm{P} 2(17.9 \pm 16.5)$ and control group $(19.6 \pm 14.1)$. For microbial growth, significant difference $(\mathrm{p}<0.05)$ for bacteria $(\mathrm{CFU} / \mathrm{ml})$ between $\mathrm{P} 1(167.7 \pm 237.1)$ and $\mathrm{P} 2$ $(240.1 \pm 321.0)$ with the control group $(464.4 \pm 448.5)$ was found. Antibiotic preparations with penicillin, streptomycin and amphotericin B on stallion semen cryopreservation reduce bacterial growth but affect post-thaw sperm motility.

Key words: bacteria, semen quality, freezing, fungi

\section{INTRODUCCIÓN}

La criopreservación de semen es un proceso fundamental para el desarrollo de biotecnologías reproductivas en equinos, dado que permite el transporte y el almacenamiento de semen a largo plazo (Loomis y Graham, 2008). Sin embargo, la calidad del semen criopreservado de equinos es aún limitada respecto a los logros alcanzados en el bovino (Liu et al., 1998), canino (Álamo et al., 2005) y porcino (Rodriguez y Wallgren, 2011). Debido a esto, se han venido buscando alternativas para el mejoramiento de los procesos de criopreservación de semen equino, principalmente a partir de los componentes incluidos en los diluyentes empleados, de tal forma que se ha evaluado la adición de diversos suplementos y crioprotectores (Palacios y Zarco, 1996; Boeta y Quintero, 2000; Squires et al., 2004; Neira et al., 2007).
Una gran variedad de bacterias comensales y potencialmente patógenas, provenientes del tracto reproductivo del macho o de la manipulación de los eyaculados durante su recolección pueden contaminar el semen equino (Althouse, 2008; Corona y Cherchi, 2009; Ortega et al., 2009). De otro lado, la calidad del semen puede disminuir debido a la competencia entre las bacterias y los espermatozoides por los nutrientes presentes en el plasma seminal, al igual que por la acumulación de catabolitos bacterianos tóxicos (Corona et al., 2006; Azawi e Ismaeel, 2012). Los microorganismos pueden causar la aglutinación de los espermatozoides móviles y la reducción en la capacidad de reacción del acrosoma, así como alteraciones en la morfología celular (Azawi e Ismaeel, 2012).

Los antibióticos y antimicóticos son componentes comúnmente utilizados en los diluyentes para la refrigeración y congelación 
del semen equino (Varner et al., 1998). Estos tienen la finalidad de eliminar la contaminación bacteriana y fúngica y reducir el riesgo de endometritis (Yániz et al., 2010; Olivieri et al., 2011). No obstante, se considera que muchos patógenos microbianos y virales pueden sobrevivir en el semen equino congelado (Corona et al., 2006; Aurich y Spergser, 2007), lo cual se suma al limitado conocimiento existente de los efectos de los antibióticos sobre los espermatozoides cuando son almacenados por largos periodos (Parlevliet et al., 2011).

Los antibióticos y antifúngicos podrían tener un efecto perjudicial sobre los espermatozoides, lo cual dificulta su empleo para la refrigeración y congelación del semen (Morrell y Wallgren, 2011; Morrell et $a l .$, 2014). La gentamicina, ampliamente utilizada en los diluyentes para semen equino, ha presentado efectos negativos en la movilidad y la velocidad de los espermatozoides durante la refrigeración y el almacenamiento prolongado (Aurich y Spergser, 2007), mientras que la ticarcilina disódica y el sulfato de amikacina, no tienen un efecto perjudicial sobre la movilidad espermática (Dietz et al., 2007). Otros antibióticos, como la estreptomicina y la penicilina sódica y potásica, han demostrado gran capacidad en el control del crecimiento de bacterias en semen equino (Varner et al., 1998), así como la anfotericina B como antimicótico durante la criopreservación (Fayrer-Hosken et al., 2008; Pillet et al., 2011). La presente investigación tuvo como objetivo evaluar el efecto de dos preparaciones antibióticas, basadas en penicilina, estreptomicina y anfotericina B, sobre la proliferación microbiana y la calidad de semen equino sometido a criopreservación.

\section{Materiales y Métodos}

\section{Material de Investigación}

Se colectó el semen de 10 caballos criollos colombianos, con edades entre 5 a 10 años, en el norte del Valle de Aburrá (Antioquia, Colombia). Los animales estuvieron sometidos a condiciones similares de alimentación, ambiente y manejo reproductivo y presentaban una condición corporal entre 5 y 6 (escala 1 a 9). Se empleó una vagina artificial modelo Missouri, lubricada con gel no espermicida y una yegua para aumentar la estimulación sexual. La fracción en gel del eyaculado fue removida con un filtro estéril. El semen de estos caballos había sido congelado con anterioridad.

En condiciones de campo, se evaluó el volumen del eyaculado mediante un tubo graduado, la movilidad espermática por microscopía óptica y la concentración seminal por espectrofotometría (Spermacue ${ }^{\circledR}$, Minitube). Solo fueron utilizados los eyaculados con mínimo de $60 \%$ de movilidad total y concentración de 100 x $10^{6}$ células $/ \mathrm{ml}$. Posteriormente, el semen fue diluido en proporción 1:1 en diluyente EquiPlus ${ }^{\circledR}$ (Minitube) $\mathrm{y}$ transportado al laboratorio en condiciones de refrigeración $\left(5^{\circ} \mathrm{C}\right)$.

\section{Suplementación con Antibióticos y Criopreservación Seminal}

La criopreservación del semen se realizó mediante un protocolo modificado de congelación (Medeiros et al., 2002; Bustamante et al., 2009). El semen fue centrifugado a $1200 \mathrm{~g}$ por $12 \mathrm{~min}$ y el precipitado fue resuspendido, para una concentración final de $100 \times 10^{6}$ espermatozoides $/ \mathrm{ml}$, en diluyente EquiPlus $^{\circledR}$ (Minitube) suplementado con 5\% de $N, N$-dimetilformamida (Sigma-Aldrich) y $5 \%$ de yema de huevo centrifugada. Dicha yema fue preparada de acuerdo a un protocolo modificado del descrito por Nouri et al. (2013), para lo cual, la yema de huevo fue diluida en una proporción de 3:1 en agua ultra pura y centrifugada a $1600 \mathrm{~g}$ durante $99 \mathrm{~min}$.

El semen fue dividido en tres alícuotas (tratamientos). La primera fue suplementada con $100 \mu \mathrm{l} / \mathrm{ml}$ de la preparación antibiótica 1 (P1), compuesta por $10000 \mathrm{UI} / \mathrm{ml}$ de penicilina y $10 \mathrm{mg} / \mathrm{ml}$ de estreptomicina (Sigma 
P4333). La segunda alícuota fue suplementada con $100 \mu \mathrm{l} / \mathrm{ml}$ de la preparación antibiótica 2 (P2), compuesta por $10000 \mathrm{UI} / \mathrm{ml}$ de penicilina, $10 \mathrm{mg} / \mathrm{ml}$ de estreptomicina y $25 \mu \mathrm{g} / \mathrm{ml}$ de anfotericina B (Sigma A5955). La tercera alícuota no recibió suplementación con antibióticos (control).

El semen fue mantenido en refrigeración a $5{ }^{\circ} \mathrm{C}$ por 20 min y luego se empacó en pajillas de $0.5 \mathrm{ml}$, mediante un equipo automático de empaque y sellado por ultrasonido (MRS1 Dual V2, IMV Technologies). Las pajillas fueron sometidas a vapores de nitrógeno líquido por $15 \mathrm{~min}$, para finalmente ser almacenadas en un tanque de almacenamiento de nitrógeno líquido (IMV Technologies).

\section{Evaluación Posdescongelación}

Cuatro pajillas por tratamiento para cada eyaculado fueron descongeladas en agua a $37{ }^{\circ} \mathrm{C}$ por $1 \mathrm{~min}$. Se evaluó la movilidad espermática mediante un procedimiento de análisis asistido por computador (CASA) (Hidalgo et al., 2005; Restrepo et al., 2013). Se utilizó un microscopio de contraste de fase (Eclipse E200, Nikon) con una cámara digital (Scout SCA780, Basler) adaptada a un computador con el software Sperm class analyser motility and concentration $\left(\mathrm{SCA}^{\circledR}\right.$, Microptic). Se analizaron los parámetros de movilidad total (MT), movilidad progresiva (MP), velocidad rectilínea (VSL), velocidad curvilínea (VCL) y velocidad media (VAP).

Se evaluó la integridad de la membrana plasmática de los espermatozoides mediante la prueba hipoosmótica (HOS) (Neild et al., 1999). Para esto, se tomaron $100 \mu l$ de semen y se adicionaron a un tubo con $500 \mu \mathrm{l}$ de una solución hipoosmótica de sacarosa 5.4\% (100 mOsmol/l). La mezcla fue incubada a $38.5^{\circ} \mathrm{C}$ por $30 \mathrm{~min}$ y luego se evaluó, mediante microscopía, la reacción (turgencia) de 200 espermatozoides en un mínimo de cinco campos de observación.

\section{Cultivo de Microorganismos del Semen}

La siembra de semen descongelado se realizó por triplicado para cada eyaculado y tratamiento. Se empleó el método de siembra en superficie con asa de vidrio en una cámara de flujo laminar. Para el cultivo de bacterias se utilizó agar nutritivo (Oxoid CM0003, Thermo Scientific), preparado en una proporción de $28 \mathrm{~g} / \mathrm{l}$. Se realizó la incubación a $37^{\circ} \mathrm{C}$ durante 48 h y el conteo de las unidades formadoras de colonias (UFC) se hizo en un contador de colonias de campo claro (Centricol). Para el cultivo de hongos se preparó agar PDA (Oxoid CM0139, Thermo Scientific) en una proporción de 39 $\mathrm{g} / \mathrm{l}$. Se realizó en cajas de petri que fueron mantenidas a temperatura ambiente $\left(22{ }^{\circ} \mathrm{C}\right.$ aprox.), durante 8 días, después de las cuales se realizó el conteo de las UFC. En ambos casos (bacterias y hongos) se cultivaron $50 \mu \mathrm{l}$ de semen en $18 \mathrm{ml}$ de agar por caja de petri.

\section{Análisis Estadístico}

Se empleó un diseño completamente al azar. Se ajustaron modelos lineales generalizados (GLM) para cada variable. Se incluyeron los efectos fijos del equino (eyaculado) y del tratamiento. Se realizó la prueba de normalidad de Shapiro Wilk y la transformación logarítmica de las variables no normales. Se calcularon coeficientes de correlación mediante la prueba de Pearson. En cada modelo, se incluyeron como covariables, los parámetros de calidad seminal con correlaciones positivas con la variable dependiente. Se realizó la comparación de medias entre los tratamientos mediante la prueba de Tukey.

\section{Resultados}

A partir de 10 eyaculados provenientes del mismo número de reproductores, se procesaron y congelaron 120 pajillas de semen (40 pajillas por tratamiento). Se encontraron 
Cuadro 1. Calidad seminal (media \pm desviación estándar) posdescongelación de semen equino, según la suplementación con antibióticos y micóticos por tratamiento (40 muestras por tratamiento)

\begin{tabular}{ccccccc}
\hline Tratamiento $^{1}$ & $\begin{array}{c}\mathrm{MT}^{2} \\
\%\end{array}$ & $\begin{array}{c}\mathrm{MP} \\
\%\end{array}$ & $\begin{array}{c}\mathrm{VCL} \\
\mu \mathrm{m} / \mathrm{s}\end{array}$ & $\begin{array}{c}\mathrm{VSL} \\
\mu \mathrm{m} / \mathrm{s}\end{array}$ & $\begin{array}{c}\mathrm{VAP} \\
\mu \mathrm{m} / \mathrm{s}\end{array}$ & $\begin{array}{c}\text { HOS } \\
\%\end{array}$ \\
\hline P1 & $34.4 \pm 19.1^{\mathrm{a}}$ & $17.9 \pm 16.5^{\mathrm{a}}$ & $70.8 \pm 23.5^{\mathrm{a}}$ & $36.6 \pm 13.6^{\mathrm{a}}$ & $48.5 \pm 20.7^{\mathrm{a}}$ & $33.0 \pm 12.8^{\mathrm{a}}$ \\
P2 & $32.2 \pm 17.6^{\mathrm{a}}$ & $15.5 \pm 14.8^{\mathrm{b}}$ & $67.9 \pm 22.4^{\mathrm{a}}$ & $33.8 \pm 14.6^{\mathrm{a}}$ & $48.4 \pm 18.9^{\mathrm{a}}$ & $31.7 \pm 13.0^{\mathrm{a}}$ \\
Control & $39.7 \pm 16.5^{\mathrm{b}}$ & $19.6 \pm 14.1^{\mathrm{a}}$ & $71.2 \pm 22.8^{\mathrm{a}}$ & $34.5 \pm 12.9^{\mathrm{a}}$ & $48.1 \pm 17.7^{\mathrm{a}}$ & $30.9 \pm 13.5^{\mathrm{a}}$ \\
\hline
\end{tabular}

${ }^{1}$ P1: Penicilina $1000 \mathrm{UI} / \mathrm{ml}$, estreptomicina $1 \mathrm{mg} / \mathrm{ml}$; P2: Penicilina $1000 \mathrm{Ul} / \mathrm{ml}$, estreptomicina $1 \mathrm{mg} / \mathrm{ml}$, anfotericina B $2.5 \mu \mathrm{g} / \mathrm{ml}$

2 MT: movilidad total; MP: motilidad progresiva; VCL: velocidad curvilínea; VSL: velocidad rectilínea; VAP: velocidad media; HOS: integridad de membrana plasmática

a,b Superíndices diferentes dentro de columnas denotan diferencia estadística significativa $(p<0.05)$

Cuadro 2. Proliferación microbiana (media \pm desviación estándar) posdescongelación de semen equino, según la suplementación con antibióticos y micóticos por tratamiento

\begin{tabular}{cccc}
\hline Tratamiento & $\mathrm{n}$ & $\begin{array}{c}\text { Bacterias } \\
\text { UFC/ml }\end{array}$ & $\begin{array}{c}\text { Hongos } \\
\text { UFC/ml }\end{array}$ \\
\hline P1 & 30 & $240.1 \pm 321.0^{\mathrm{a}}$ & $1.7 \pm 1.0^{\mathrm{a}}$ \\
P2 & 30 & $167.7 \pm 237.1^{\mathrm{a}}$ & $1.5 \pm 0.8^{\mathrm{a}}$ \\
Control & 30 & $464.4 \pm 448.5^{\mathrm{b}}$ & $1.7 \pm 0.8^{\mathrm{a}}$ \\
\hline
\end{tabular}

${ }^{1}$ P1: Penicilina $1000 \mathrm{UI} / \mathrm{ml}$, estreptomicina $1 \mathrm{mg} / \mathrm{ml}$; P2: Penicilina $1000 \mathrm{Ul} / \mathrm{ml}$, estreptomicina $1 \mathrm{mg} / \mathrm{ml}$, anfotericina B $2.5 \mu \mathrm{g} / \mathrm{ml}$

${ }^{a, b}$ Superíndices diferentes dentro de columnas denotan diferencia estadística significativa $(p \varangle 0.05)$

coeficientes de variación entre 20 y $40 \%$ para VCL, VSL, VAP y HOS, entre 40 y $60 \%$ para MT, HOS y proliferación de hongos, y mayores de 60\% para MP y proliferación de bacterias. El efecto individual del equino (eyaculado) fue significativo $(\mathrm{p}<0.05)$ para todas las variables.

El Cuadro 1 presenta los resultados de los parámetros de calidad seminal por trata- miento. La MT fue estadísticamente mayor para el grupo control respecto a las preparaciones antibióticas 1 y $2(\mathrm{p}<0.05)$, mientras que la MP fue menor en el tratamiento P2 con relación a P1 y al grupo control $(\mathrm{p}<0.05)$. Por otro lado, no se halló diferencia estadística entre tratamientos para los parámetros de cinemática de los espermatozoides (VCL, VSL y VAP) y para la integridad de la membrana plasmática (HOS). 


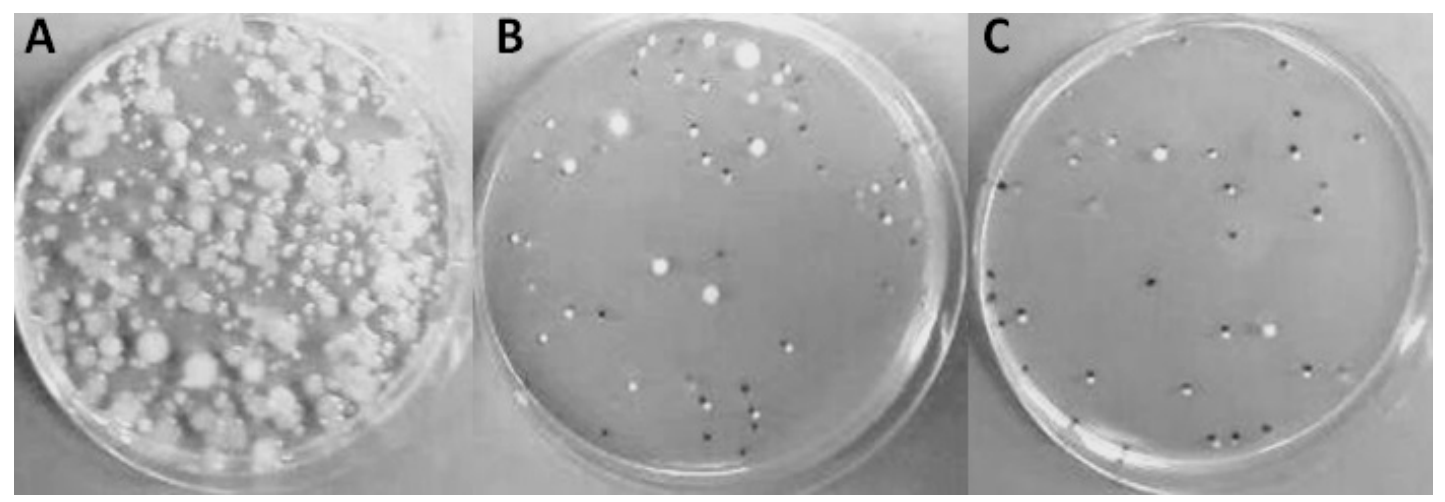

Figura 1. Siembra de semen de equino descongelado mostrando la proliferación de bacterias en agar nutritivo: A: grupo control (sin antibiótico). B: Preparación antibiótica 1 (Penicilina 1000 UI/ml, estreptomicina $1 \mathrm{mg} / \mathrm{ml}$ ); C: Preparación antibiótica 2 (Penicilina 1000 UI/ $\mathrm{ml}$, estreptomicina $1 \mathrm{mg} / \mathrm{ml}$, anfotericina B $2.5 \mu \mathrm{g} / \mathrm{ml}$ )

Se observó una reducción en la proliferación de bacterias por efecto de las preparaciones antibióticas 1 y 2 , respecto al control, pero sin diferencias estadísticas entre las dos preparaciones ( $\mathrm{p}<0.05$; Cuadro 2). Por otro lado, no hubo diferencias en la proliferación de hongos por efecto de las preparaciones antibióticas 1 y 2 con el grupo control.

La Figura 1 muestra la proliferación de colonias de bacterias de muestras seminales descongeladas que fueron cultivadas en agar nutritivo. Los coeficientes de correlación entre la proliferación bacteriana y los parámetros de calidad seminal MT, MP y HOS fueron de $-0.29,-0.21$ y -0.45 , respectivamente $(\mathrm{p}<0.05)$.

\section{Discusión}

La calidad del semen equino criopreservado es fundamental para la obtención por inseminación artificial de gestaciones a término. Los resultados obtenidos para los diferentes parámetros de calidad seminal (Cuadro 1) pueden considerarse similares a reportes previos en caballos criollos colombianos (Restrepo et al., 2013). El efecto individual del reproductor (eyaculado) podría explicar gran parte de la variabilidad encontrada para todas las variables $(\mathrm{p}<0.05)$. Diversos reportes muestran una amplia proporción de la variabilidad espermática, especialmente en parámetros como la movilidad progresiva, vitalidad e integridad de membrana debida al reproductor y al eyaculado (Vidament et al., 1998).

La mayoría de los eyaculados recolectados de animales sanos se encuentran contaminados con microorganismos (Kristula y Smith, 2004; Morrell y Wallgren, 2011), los cuales podría esperarse que provengan de la mucosa del prepucio y la uretra (Pasing et $a l .$, 2013). En el semen equino se ha reportado una moderada incidencia de los géneros Corynebacterium y Staphylococcus (coagulasa-negativos) y de la clase Actinobacteria. De igual forma, se reporta una baja incidencia de microorganismos de la familia Enterobacteriacae, de los géneros Micrococcus, Bacillus, Streptomyces y Streptococcus (alfa-hemolíticos) y de las bacterias Escherichia coli, Klebsiella pneumoniae, Pseudomonas aeruginosa y Streptococcus equi, entre otras (Madsen y Christensen, 1995; Dirscherl, 2011; Pasing et al., 2013). Entre los mohos, se ha reportado el Aspergillus sp y entre las levaduras Candida albicans (Vaid et al., 2012). 
Las bacterias pueden disminuir la viabilidad de las células espermáticas a través de mecanismos asociados a la apoptosis, como la excesiva producción de especies reactivas de oxígeno (ERO) y la activación de caspasas (Villegas et al., 2005; Olivieri et al., 2011; Rodriguez y Wallgren, 2011). Los coeficientes de correlación negativos, encontrados en el presente estudio, entre la proliferación de bacterias y algunos parámetros de calidad seminal, corroboran el efecto deletéreo de las bacterias sobre la calidad seminal. Sin embargo, la reducción observada en la calidad seminal también estuvo asociada a la suplementación con antibióticos, toda vez que se encontró una reducción en MT y MP por la adición de P1 y P2, y por la adición de P2, respectivamente (Cuadro 1).

Jasko et al. (1993) encontraron que los antibióticos sulfato de amikasina, ticarcilina disódica y sulfato de gentamicina, en concentraciones mayores a $1000 \mathrm{UI} / \mathrm{ml}$, afectaban los parámetros de movilidad del semen equino refrigerado. El efecto deletéreo de algunos antibióticos sobre la calidad del semen equino también ha sido demostrado en otros estudios (Varner et al., 1998; Aurich y Spergser, 2007). Incluso se ha llegado a la implementación de otras estrategias, como la remoción de bacterias por centrifugación (Morrell y Wallgren, 2011; Morrell et al., 2014).

Los indicadores de la cinemática de los espermatozoides (VSL, VCL y VAP), al igual que la integridad de la membrana plasmática (HOS), no se vieron alterados por efecto de la adición de antibióticos (Cuadro 1). No obstante, Varner et al. (1998) encontraron que la combinación de penicilina $\mathrm{G}$ y amikacina indujo porcentajes de movilidad, VCL, VSL y VAP, superiores al uso individual de estos antibióticos, mientras la polimixina B presentó valores inferiores de movilidad y cinemática, incluso en comparación con la gentamicina, la estreptomicina y la penicilina sódica y potásica.
El uso de antibióticos en el semen equino ha dado como resultado un aumento positivo de la longevidad de los espermatozoides y de su movilidad (Dietz et al., 2007). Muchos de los diluyentes para semen utilizados en la actualidad contienen antibióticos para disminuir el crecimiento de bacterias (Klein et al., 2010); asimismo, se acepta la suplementación con antimicóticos (Olivieri et al., 2011). Sin embargo, algunos estudios indican que los antibióticos añadidos al medio crioprotector no son adecuados para proteger el semen congelado de la contaminación por bacterias y hongos (Corona et al., 2006; Althouse, 2008).

Los resultados de la presente investigación demuestran que el uso de dos preparaciones antibióticas, basadas en penicilina, estreptomicina y anfotericina $\mathrm{B}$, tiene un marcado efecto de reducción de la carga de bacterias del semen equino congelado y descongelado (Cuadro 2). Asimismo, la alta proliferación de colonias de bacterias en el grupo control (Figura 1), muestra su alto nivel de criotolerancia (Corona y Cherchi, 2009).

No se observó una reducción significativa sobre la proliferación de hongos, lo cual podría atribuirse a la concentración de anfotericina B $(2.5 \mu \mathrm{g} / \mathrm{ml})$ o a su espectro de acción antimicótica. Fayrer-Hosken et al. (2008) emplearon una concentración de 50 $\mu \mathrm{g} / \mathrm{ml}$, mientras Pillet et al. (2011) reportan el uso de $0.25 \mu \mathrm{g} / \mathrm{ml}$. Esto evidencia la ausencia de claridad sobre la concentración de suplementación más apropiada. Los trabajos que evalúan el uso de antimicóticos en semen equino son escasos.

Se concluye que el uso de preparaciones antibióticas de penicilina, estreptomicina y anfotericina $\mathrm{B}$, en la congelación de semen equino, constituye un método de control efectivo de la proliferación bacteriana posdescongelación. Es así que se podría reducir los riesgos de infecciones en las yeguas, a causa de la inseminación artificial con semen congela- 
do contaminado; sin embargo, se requiere continuar con la investigación de alternativas de control antimicrobiano en el semen criopreservado, dada la reducción en la calidad espermática generada por el uso de antibióticos.

\section{Literatura Citada}

1. Álamo D, Batista M, González F, Rodríguez N, Cruz G, Cabrera F, Gracia A. 2005. Cryopreservation of semen in the dog: use of ultra-freezers of $-152^{\circ} \mathrm{C}$ as a viable alternative to liquid nitrogen. Theriogenology 63: 72-82. doi: 10.1016/ j.theriogenology.2004.03.016

2. Althouse GC. 2008. Sanitary procedures for the production of extended semen. Reprod Dom Anim 43(Suppl 2): 374-378. doi: 10.1111/j.14390531.2008.01187.x

3. Aurich C, Spergser J. 2007. Influence of bacteria and gentamicin on cooledstored stallion spermatozoa. Theriogenology 67: 912-918. doi: 10.1016/ j.theriogenology.2006.11.004

4. Azawi O, Ismaeel M. 2012. Effects of seasons on some semen parameters and bacterial contamination of Awassi ram semen. Reprod Dom Anim 47: 403-406. doi: 10.1111/j.1439-0531.2011.01888.x

5. Boeta M, Quintero L. 2000. Utilización de leche descremada ultrapasteurizada como diluyente de semen refrigerado de burro, destinado a la inseminación de yeguas. Vet Méx 31: 67-69.

6. Bustamante I, Pederzolli C, Sgaravatti A, Gregory R, Dutra C, Jobim M, Mattos R. 2009. Skim milkegg yolk based semen extender compensates for non-enzymatic antioxidant activity loss during equine semen cryopreservation. Anim Reprod 6: 392399.

7. Corona A, Cassu I, Bertulu A, Cherchi $R$. 2006. Characterisation of bacteria in fresh semen of stallions during the breeding season. Anim Reprod Sci 94:
85-88. doi: 10.1016/j.anireprosci. 2006.04.022

8. Corona A, Cherchi R. 2009. Microbial quality of equine frozen semen. Anim Reprod Sci 115: 103-109. doi: 10.1016/ j.anireprosci.2008.11.016

9. Dietz JP, Sertich PL, Boston RC, Benson CE. 2007. Comparison of ticarcillin and piperacillin in Kenney's semen extender. Theriogenology 68: 848852. doi: $10.1016 /$ j.theriogenology. 2007.03.031

10. Dirscherl FKM. 2011. Effects of semen collection practices on the bacterial load of stallion semen. PhD Thesis. Germany: University of Veterinary Medicine Hannover. $110 \mathrm{p}$.

11. Fayrer-Hosken R, Abreu-Barbosa C, Heusner G Jones L. 2008. Cryopreservation of stallion spermatozoa with INRA96 and glycerol. J Equine Vet Sci 28: 672-676. doi: 10.1016/j.jevs. 2008.10.006

12. Hidalgo M, Rodriguez I, Dorado J, Sanz J, Soler C. 2005. Effect of sample size and staining methods on stallion sperm morphometry by the Sperm Class Analyzer. Vet Med-Czech 50: 24-32.

13. Jasko DJ, Bedford SJ, Cook NL, Mumford EL, Squires EL, Pickett BW. 1993. Effect of antibiotics on motion characteristics of cooled stallion spermatozoa. Theriogenology 40: 885893.

14. Klein C, Donahue JM, Sells SF, Squires EL, Timoney PJ, et al. 2010. Antibiotic-containing semen extender reduces the risk of transmission of CEM. Anim Reprod Sci 121: 222-223. doi: 10.1016/j.anireprosci.2010.04.169

15. Kristula MA, Smith BI. 2004. Diagnosis and treatment of four stallions, carriers of the contagious metritis organism - case report. Theriogenology 61: 595-601. doi: 10.1016/S0093$691 X(03) 00228-0$

16. Liu Z, Foote R, Brockett C. 1998. Survival of bull sperm frozen at different rates in media varying in osmolarity. 
Cryobiology 37: 219-230. doi: 10.1006/ cryo.1998.2117

17. Loomis P, Graham J. 2008. Commercial semen freezing: individual male variation in cryosurvival and the response of stallion sperm to customized freezing protocols. Anim Reprod Sci 105: 119-128. doi: 10.1016/j.anireprosci. 2007.11.010

18. Madsen M, Christensen P. 1995. Bacterial flora of semen collected from Danish warmblood stallions by artificial vagina. Acta Vet Scand 36: 1-7.

19. Medeiros A, Gomes G, Carmo M, Papa F, Alvarenga M. 2002. Cryopreservation of stallion sperm using different amides. Theriogenology 58: 273-276. doi: 10.1016/S0093-691X(02) 00898-1

20. Morrell JM, Klein C, Lundeheim N, Erol E, Troedsson MH. 2014. Removal of bacteria from stallion semen by colloid centrifugation. Anim Reprod Sci 145: 47-53. doi: 10.1016/j.anireprosci.2014.01.005

21. Morrell JM, Wallgren M. 2011. Removal of bacteria from boar ejeculates by single layer centrifugation can reduce the use of antibiotics in semen extender. Anim Reprod Sci 123: 6469. doi: 10.1016/j.anireprosci.2010.11.005

22. Neild D, Chaves G Flores M, Mora N, Beconi M, Agüero A. 1999. Hypoosmotic test in equine spermatozoa. Theriogenology 51: 721-727. doi: 10.1016/S0093-691X(99)00021-7

23. Neira JA, Ramírez GF, León SA, Moreno $D A .2007$. Efecto de la asociación L-glutamina-etilenglicol en la criopreservación de semen equino. Rev Med Vet 14: 93-105.

24. Nouri H, Towhidi A, Zhandi M, Sadeghi R. 2013. The effects of centrifuged egg yolk used with INRA plus soybean lecithin extender on semen quality to freeze miniature Caspian horse semen. J Equine Vet Sci 33: 1050-1053. doi: 10.1016/j.jevs.2013.03.184

25. Olivieri B, Love B, Rezabek G, Lamm C, Vamer D, Payton M, Reed G 2011. Effect of antibiotic-containing extender on Taylorella equigenitalis contaminated semen. J Equine Vet Sci 31: 655-660. doi: 10.1016/j.jevs.2011.04.002

26. Ortega C, González L, Muriel A, Macías B, Rodríguez H, Tapia J. 2009. Does the microbial flora in the ejaculate affect the freezeability of stallion sperm? Reprod Dom Anim 44: 518-522. doi: 10.1111/j.1439-0531. 2008.01267.x

27. Palacios A, Zarco L. 1996. Efecto de la sustitución de yema de huevo por albúmina sérica bovina, suero equino o suero bovino en el diluyente de congelación sobre la viabilidad pos-descongelación del espermatozoide equino. Vet Méx 27: 221-227.

28. Parlevliet J, Lynn J, Paccamonti D. 2011. The use of cefquinome in equine semen extender. J Equine Vet Sci 31: 139-142. doi: 10.1016/j.jevs.2010.12.015

29. Pasing SS, Aurich C, von Lewinski M, Wulf M, Kruger M, Aurich JE. 2013. Development of the genital microflora in stallions used for artificial insemination throughout the breeding season. Anim Reprod Sci 139: 53-61. doi: 10.1016/ j.anireprosci.2013.03.009

30. Pillet E, Duchamp G, Batellier F, Beaual V, Anton M, Desherces S, et al. 2011. Egg yolk plasma can replace egg yolk in stallion freezing extenders. Therioge-nology 75: 105-114. doi: 10.1016/j.theriogenology.2010.07.015

31. Restrepo G, Ocampo D, Velásquez A. 2013. Evaluación de la movilidad del semen criopreservado de caballo criollo colombiano por un sistema analizador de clase. Rev UDCA 16: 445-450.

32. Rodriguez H, Wallgren M. 2011. Advances in boar semen cryopreservation. Vet Med Int 2011: Art ID 396181. doi: 10.4061/2011/396181

33. Squires E, Keith S, Graham J. 2004. Evaluation of alternative cryoprotectants for preserving stallion spermatozoa. Theriogenology 62: 1056-1065. doi: 10.1016/j.theriogenology.2003.12.024

34. Vaid R, Arangasamy A, Talluri T, Ravi $S$, Bera B, Anand T, Riyesh T, et al. 2012. Microbiological quality of fresh 
and frozen equine semen of Indian horses. Vet Practitioner 13: 336-339.

35. Varner D, Scanlan C, Thompson J, Brumbaugh $G$ Blanchard T, Carlton C, Johnson L. 1998. Bacteriology of preserved stallion semen and antibiotics in semen extenders. Theriogenology 50: 559-573. doi: 10.1016/S0093691X(98)00161-7

36. Vidament M, Cognard E, Yvon J-M, Sattler M, Palmer E, Magistrini M. 1998. Evaluation of stallion semen before and after freezing. Reprod Dom
Anim 33: 271-277. doi: 10.1111/j.14390531.1998.tb01357.x

37. Villegas J, Schulz M, Soto L, Sanchez R. 2005. Bacteria induce expression of apoptosis in human spermatozoa. Apoptosis 10: 105-110. doi: 10.1007/ s 10495-005-6065-8

38. Yániz J, Marco-Aguado M, Mateos J, Santolaria P. 2010. Bacterial contamina-tion of ram semen, antibiotic sensitivities, and effects on sperm quality during storage a $15{ }^{\circ} \mathrm{C}$. Anim Reprod Sci 122 : 142-149. doi: 10.1016/j.anireprosci.2010.08.006 